\title{
Independent component analysis and nongaussianity for blind image deconvolution and deblurring
}

\author{
Hujun Yin and Israr Hussain \\ School of Electrical and Electronic Engineering, The University of Manchester, Manchester M60 1QD, UK \\ E-mail: h.yin@manchester.ac.uk, i.hussain@postgrad.manchester.ac.uk
}

\begin{abstract}
Blind deconvolution or deblurring is a challenging problem in many signal processing applications as signals and images often suffer from blurring or point spreading with unknown blurring kernels or point-spread functions as well as noise corruption. Most existing methods require certain knowledge about both the signal and the kernel and their performance depend\$ on the amount of prior information regarding the both. Independent component analysis (ICA) has emerged as a useful method for recovering signals from their mixtures. However, ICA usually requires a number of different input signals to uncover the mixing mechanism. In this paper, a blind deconvolution and deblurring method is proposed based on the nongaussianity measure of ICA as well as a genetic algorithm. The method is simple and does not require prior knowledge regarding either the image or the blurring process, but is able to estimate or approximate the blurring kernel from a single blurred image. Various blurring functions are described and discussed. The proposed method has been tested on images degraded by different blurring kernel\$ and the results are compared to those of existing methods such as Wiener filter, regularization filter, and the Richardson-Lucy method. Experimental results show that the proposed method outperform these methods.
\end{abstract}

\section{Introduction}

Signals and images often suffer from degradation due to imperfection in the capturing and imaging process; therefore a recorded signal/image is inevitably a degraded version of the original signal/image. For instance, recorded neuronal activities (action potentials or local field potentials) are often mixed activities of several neurons or a population; satellite/aerial images usually suffer from degradation from atmospheric turbulence and lens defocusing. In addition to these blurring effects, noise may corrupt any recorded image/signal. It can be introduced into the system by the creating medium or by the recording medium or simply because of the measurement precision. Undoing these imperfections is crucial for many image processing and multidimensional signal processing tasks. For most signal reconstruction methods, the characteristics of the degrading system and the noise need to be known or are often assumed to be known a priori. Then the restoration process may become straightforward or even trivial. However, this information is not always available for various reasons. Therefore the reconstruction requires not only restoration of the image/signal but also estimation of the blurring parameters from the degraded image/signal - the procedure is often referred to as blind deconvolution.

Blind deconvolution is not a new area and there exist several approaches and methods $[1,2,4,8]$. For instance, an automatic image deblurring algorithm based on an adaptive Gaussian model has been proposed and it has been shown robust and to yield satisfactory results on high resolution satellite data [7]. However, it is limited in the sense that it is based on Gaussian models and works mainly on satellite images. In [11] an algorithm for blind restoration of blurred and noisy images was proposed; the technique involves two processing blocks, one for denoising using the singular value decomposition and compression filtering, and one for deblurring based on a double regularization technique. It has been demonstrated that this technique is effec- 
tive in restoring severely blurred and noise-corrupted images without exact knowledge of either the noise or image characteristics. This method, however, is not completely blind. It requires certain prior information on the image degradation function to implement the algorithm. An exhaustive list of typical methods for image restoration and identification can be found in [9]; a few more methods such as the Richardson-Lucy and its variants are described in $[3,10,13,16]$. These previous techniques are limited in the sense that they are either domain specific, i.e. for a particular category of images or particular type of degrading functions, or require certain prior knowledge about the point spread function (PSF), the image and/or the noise.

The motivation of this work is to derive an effective and efficient method based on nongaussianity measures of independent component analysis (ICA), in order to perform a completely blind deblurring or deconvolution with or without the presence of noise. ICA has been predominantly used for 1-D signals such as speech and EEG signal processing; and it usually requires multiple observed signals. Applying to blind deconvolution, especially on a single blurred image/signal is a challenging task. A recent approach has been proposed in [14]. It applies ICA on neighbouring shifted sub images to extract the original images and its derivatives [14]. However, the assumption that the original image and its derivatives are statistically independent may not be valid, especially when blurring is medium to severe. Experimental results of only weak blurring have been reported. In this paper a different approach is proposed using ICA principle and associated nongaussianity measures. The proposed method requires no prior knowledge about image, noise and blurring functions and is based on single blurred image. Although this paper focuses on deblurring image, the proposed blind deconvolution method is applicable to other signals in general. A preliminary result on 1-D signals and images has been reported in [17]. There exist many types of degradation function such as misfocus, motion, or atmospheric turbulence. The proposed method can cater to the degradation caused by any kind of blurring function.

The layout of the paper is as follows. The concepts related to ICA and nongaussianity measures for deconvolution and deblurring are explained in the next Section. Then in Section 3, the proposed method is described in detail with the experimental setups, together with the results on various degradation functions and comparisons with the existing methods. The discussions and conclusions are given in Section 4.

\section{ICA and Nongaussianity for Deblurring}

A blurred image can be modelled as the convolution of an original image with a 2-D blurring kernel or pointspread function (PSF) with or without a noise term,

$$
\begin{gathered}
x(i, j)=f(i, j) \otimes b(i, j)+\eta(i, j)=\sum_{k=-m}^{m} \sum_{l=-n}^{n} \\
b(k, l) f(i+k, j+l)+\eta(i, j),
\end{gathered}
$$

where $\otimes$ denotes the convolution operator, $x(i, j)$ is the degraded image, $f(i, j)$ is the original image; $b(k$, $l)$ is the PSF; $\eta(i, j)$ is the added noise; and $m$ and $n$ are the effective ranges of the blurring kernel on $i$ and $j$ axes, respectively.

The PSFs under consideration and experimented here are not functions of spatial location; in other words, they are spatially invariant, though the proposed technique can, in principle, be extended to deal with spatially variant blurring. Essentially, this means that the image is blurred in exactly the same way at every spatial location.

In blind deconvolution, a convoluted version $x$ of an original signal $f$ is observed, without knowing either the signal $f$ or the convolution kernel $b[12,14]$. The problem is then to find a deblurring filter $h$ so that $f=h \otimes x$; this is often an ill-posed mathematical problem. The equalizer or deblurring filter $h$ is assumed to be or realized as a FIR filter of sufficient length, so that the truncation effect can be ignored. A special case of blind deconvolution that is especially interesting is the case where it is assumed that the values of the signal $f$ at two different points of space are statistically independent. Under certain assumptions, this problem can be solved by simply whitening the observed signal $x$. However, to solve the problem in full, one must assume that the signal $f$ is non-Gaussian, and use higher-order information [5,6]. Thus the techniques used for solving this special case of the problem are very similar to those used in other higher-order methods like ICA. ICA is a technique that recovers a set of independent signals from a set of observed mixed signals without $a$ priori knowledge of the sources, and is also closely associated to blind source separation (BSS). It is assumed that each observed signal is a linear combination of the independent source signals, and that the mixing mechanism is generally not known. The independency of the sources makes it possible for subsequent application of ICA to recover original source signals.

Further, the distributions of the source signals are assumed to be unknown, except that they may not be 
Gaussian, or at most only one source can be Gaussian. Basic working methodology behind such a scheme is to look for nongaussianity in the recovered signals. There is a clear link between ICA and blind deconvolution; more precisely blind deconvolution is a special case of ICA where the original signals are i.i.d over space (or time in 1-D case). In blind deconvolution, we have only one observed signal (output) and one source (input). The observed signal consists of an unknown source signal mixed with itself at different spatial locations (or time delays in 1D case). This could be due to echoes caused by the reverberating nature of the environment or degradations as mentioned before. The task is to estimate the source signal from the observed signal only, without knowing the mixing or convolving system, the time delays, or the mixing coefficients.

From the Central Limit Theorem (CLT), we know that the output of a linear system tends to be more Gaussian. ICA tries to find a solution that maximizes the "nongaussianity" of the recovered signal. Because of that, the technique does not usually work with Gaussian signals, since their higher cumulants are equal to zero. The CLT implies that if we can find a combination of the measured signals with minimal Gaussian properties, then that signal will be one of the independent signals. In order to do this a way to measure nongaussianity is required.

Kurtosis is a classical method for measuring nongaussianity. When data is pre-processed to have unit variance, kurtosis is related to the fourth moment of the data. In an intuitive sense, kurtosis measures how "spikiness" of a distribution or the size of the tails. Kurtosis is simple to calculate; however, it is sensitive to outliers in the data set. Its values may be dominated by only few values in the tails, which means that its statistical significance is poor. Mathematically kurtosis is defined as

$$
\operatorname{Kurt}(y)=E\left\{y^{4}\right\}-3\left(E\left\{y^{2}\right\}\right)^{2} .
$$

In cases where $y$ has unit variance, $\operatorname{Kurt}(y)=$ $E\left\{y^{4}\right\}-3$. In other words, kurtosis is a normalized version of the fourth moment. It is zero for Gaussian variables and is greater or smaller than zero for superor sub-Gausian variables, respectively. Often the absolute value of kurtosis is used to measure the nongaussianity. As kurtosis is sensitive to outliers and noise, in many situations there is need for a more robust measure of nongaussianity. Negentropy is the one and is often used for this purpose. Unlike kurtosis, the negentropy is robust but computationally complex. In practice it can only be approximated.
Negentropy is based on the information theoretic quantity of differential entropy, or simply entropy as it is often called. The entropy of a random variable is related to the information that the observation of the variable gives. The more unpredictable and unstructured the variable is, the larger the entropy. As a result Gaussian variable has the largest entropy; all other structured or clustered data sets have entropy less than that of the Gaussian. This very fact can be used as a measure of nongaussianity.

A normalized version of differential entropy is used for obtaining a measure of nongaussianity that is zero for Gaussian variable and always nonnegative for others. This is defined as 'negentropy', which can be stated as

$$
J(Y)=H\left(Y_{\text {gauss }}\right)-H(Y)
$$

where $H(Y)$ is the (differential) entropy of the variable $Y$ and $H\left(Y_{\text {gauss }}\right)$ is the (differential) entropy of the Gaussian variable of the same correlation (covariance) matrix as $Y$. Negentropy is always nonnegative and is zero for Gaussian variables. It is a robust measure of nongaussianity; however the problem is in its estimation for which one requires knowledge of the pdf of the given variable. Therefore some approximation techniques will have to be used for estimating negentropy. A typical method is to use higher-order cumulants, or polynomial expansions. This gives,

$$
J(y) \approx \frac{1}{12} E\left\{y^{3}\right\}^{2}+\frac{1}{48} k u r t(y)^{2},
$$

where $y$ is assumed to be zero mean and unit variance. However, the problem with this approximation is that one again relies on kurtosis for measuring the nongaussianity. As it can be seen that the first term in Eq. (4) under symmetric distributions is zero, therefore one is left with square of kurtosis. Hence it is prone to the same problems of kurtosis. More sophisticated techniques for approximation of negentropy are required.

One way could be, to use expectations of general non-quadratic functions or non-polynomial moments, to replace polynomial functions ' $y$ ' ' and ' $y$ ' ' by other functions possibly two or more. This gives a simple way of approximating negentropy. For a simple case the approximation becomes [6]

$$
\begin{aligned}
J(y) \approx & k_{1}\left(E\left\{G_{o}(y)\right\}\right)^{2}+k_{2}\left(E\left\{G_{e}(y)\right\}\right. \\
& \left.-E\left\{G_{e}(v)\right\}\right)^{2},
\end{aligned}
$$

where $k_{1}$ and $k_{2}$ are positive constants; $v$ is Gaussian variable and both $y$ and $v$ are of zero mean and unit variance; $G_{0}$ and $G_{e}$ are odd and even nonquadratic 

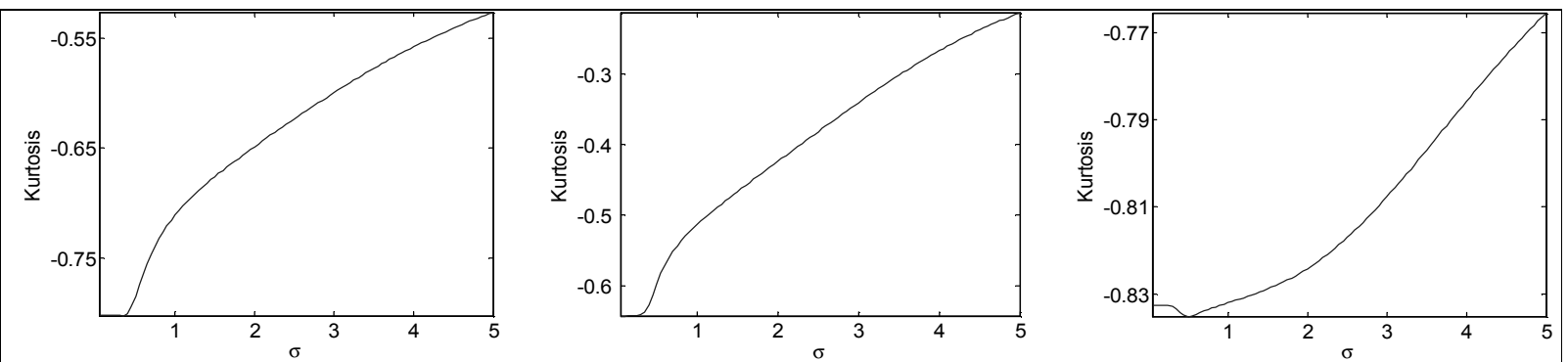

Fig. 1. Nongaussianity analysis for Barbra (left), Lena (middle) and Cameraman (right) images for increased blurring.
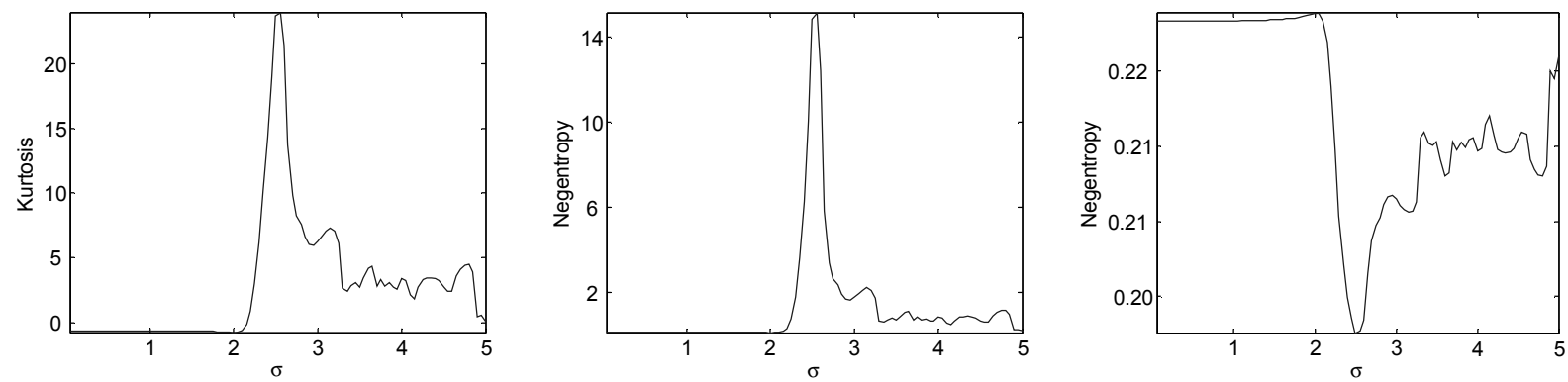

Fig. 2. Nongaussianity analysis on deblurring Barbra image (kurtosis: Eq. (2)-left, negentropy: Eq. (4)- middle, and negentropy: Eq. (5) with functions of Eqs (6) and (7) - right).

functions respectively. This measure is more robust than the one in Eq. (4). Different choices exist for nonquadratic functions. A useful choice is [6],

$$
\begin{aligned}
& G_{o}(y)=\frac{1}{a_{1}} \log \cosh \left(a_{1} y\right), \\
& G_{e}(y)=-\exp \left(-\frac{y^{2}}{2}\right),
\end{aligned}
$$

where $1 \leqslant a_{1} \leqslant 2$. Thus one can obtain the negentropy that is a good compromise between the properties of two classic nongaussianity measures given by kurtosis and negentropy.

The above mentioned measures can be applied for finding independency and nongaussianity in the subsequent deblurring process. The observed signals or images need to be centred and normalized before applying these measures. The blurring process produces correlation between the adjacent points of the signal thus making the signal/image more Gaussian than the original. Further, the stronger or wider the blurring the more the degraded signal/image moves towards Gaussian. Figure 1 illustrates this very fact where the nongaussianity measure of Eq. (2) was taken on blurred images (with a Gaussian kernel of varying width, $\sigma$ ) of Barbra, Lena and Cameraman respectively. The images become more Gaussian as the results of blur- ring and furthermore the stronger the blurring the more Gaussian the images become.

As stated earlier, the CLT implies that the output of a linear system is more Gaussian than the original signal. Therefore if one wishes to recover the original signal, it would be the one that has the minimum Gaussian properties. Therefore a filter that maximizes the nongaussianity of the mixed/degraded signal would be the inverse filter (if it exists) of the original blurring filter. An optimization process can be performed on these nongaussianity measures. Then adaptive learning rules can be obtained for estimating iteratively the deblurring filter parameters $W$, the inverse of the blurring filter. Following [6], a gradient-based learning algorithms can be obtained based on either kurtosis or negentropy measure,

$$
\begin{aligned}
& \Delta W \propto \operatorname{sign}(K \operatorname{Kurt}(W \otimes X)) E\left\{X(W \otimes X)^{3}\right\}, \\
& \Delta W \propto(E\{G(W \otimes X)\}-E\{G(v)\}) \\
& \quad E\{X g(W \otimes X)\},
\end{aligned}
$$

where $G$ is a nonquadratic function and $g$ is the derivative of $G$ and $v$ is a standardized Gaussian random variable. Eq. (9) is derived from a simplified case of negentropy measure of Eq. (5), where only the second term is considered. 

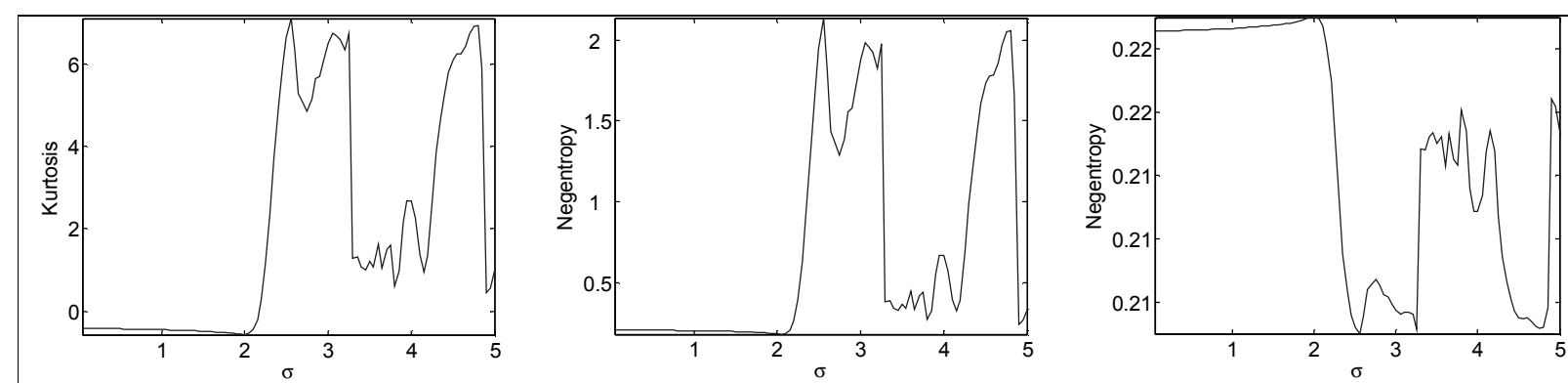

Fig. 3. Nongaussianity analysis on deblurring Cameraman image (left - kurtosis: Eq. (2); middle - negentropy: Eq. (4); and right - negentropy: Eq. (5) with functions of Eqs (6) and (7)).
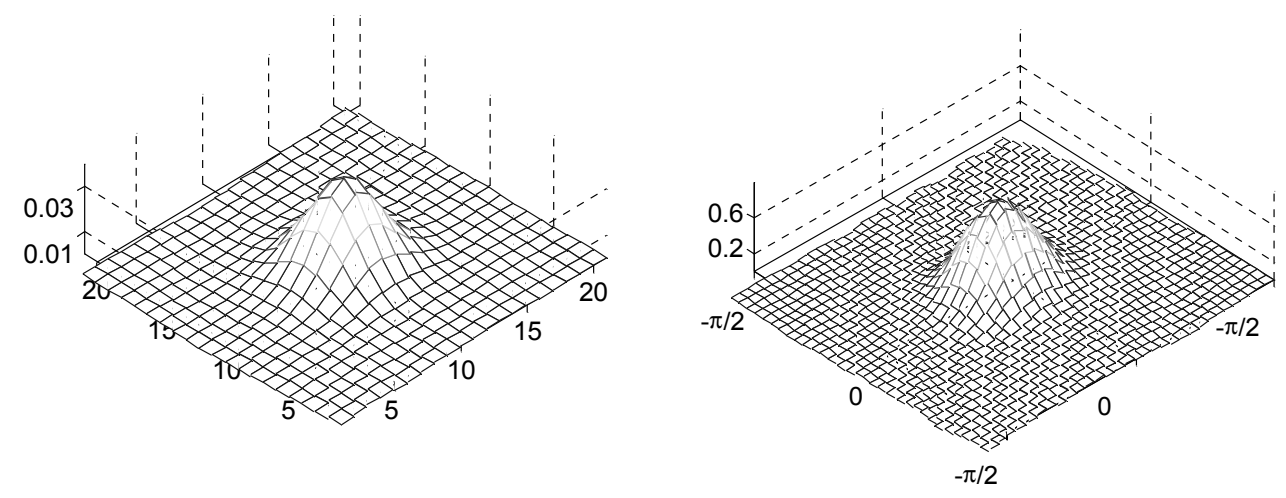

Fig. 4. Gaussian PSF (with $\sigma=2$ ) and its Fourier transform (magnitude).

Figures 2 and 3 show various nongaussianity measures of a deblurring system on two images (Barbra and Cameraman). Both images have been blurred with a Gaussian PSF. These measures are kurtosis Eq. (2), and negentropy Eqs (4) and (5) respectively, on the deblurred images of various deblurring kernel width.

It is worthwhile to mention here that the above nongaussianity analysis is not only true for Gaussian blurring filter but also for other point-spread functions, such as uniform out of focus blur, atmospheric blur or motion blur (see next section). Furthermore, nongaussianity analysis can be used to find and optimize the blurring filter and its parameters, e.g. Eqs (8) and (9), thus paving the way for estimation of the degrading filter and thus the blind deconvolution of the signal. However as shown in Figs 2 and 3, the gradient based methods can be easily trapped in local minima.

\section{Proposed method and experimental results}

In this Section, we present a blind image deconvolution and deblurring approach based on nongaussianity measures and genetic algorithms. The method makes use of the difference between blurred (correlated) and un-blurred (original) images, using the very fact of the CLT. Therefore the original image can be recovered using a nongaussianity measure as ICA and BSS use to find independent components and to separate the signals. Here the nongaussianity measure is used to differentiate between the correlated, blurred image and the uncorrelated image; the restored image is more independent (or uncorrelated) than the blurred one. The blurring type and parameters of the estimated PSF are optimized a genetic algorithm in an iterative manner. The operation is conducted in the spatial frequency domain. The approach is computationally simple and efficient. The process can be summarized in the following steps:

1. Initialize the genetic algorithm parameters: encoding bits (e.g. 16 bits), population size (e.g. 2000), crossover rate (e.g. 0.25), mutation rate $(0.005)$, etc.

2. Calculate the nongaussianity of the blurred image and Fourier coefficients of the blurred image.

3. Perform one iteration for different types and values of the blurring kernel parameters; find the restored image through inverse/wiener filtering in the spectral domain based on the current estimate 

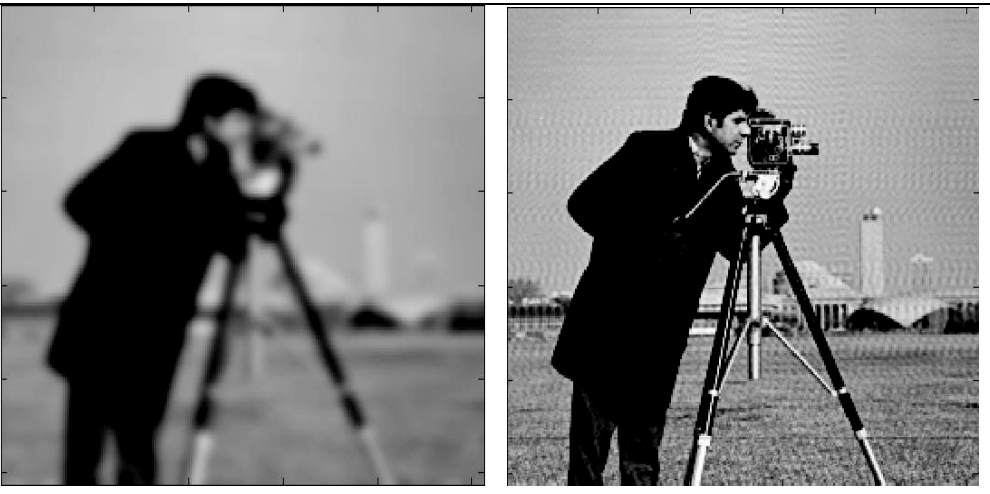

Fig. 5. Degraded Cameraman image with Gaussian blurring $(\sigma=3)$ and restored with the proposed method (estimated $\sigma=2.91$ ).
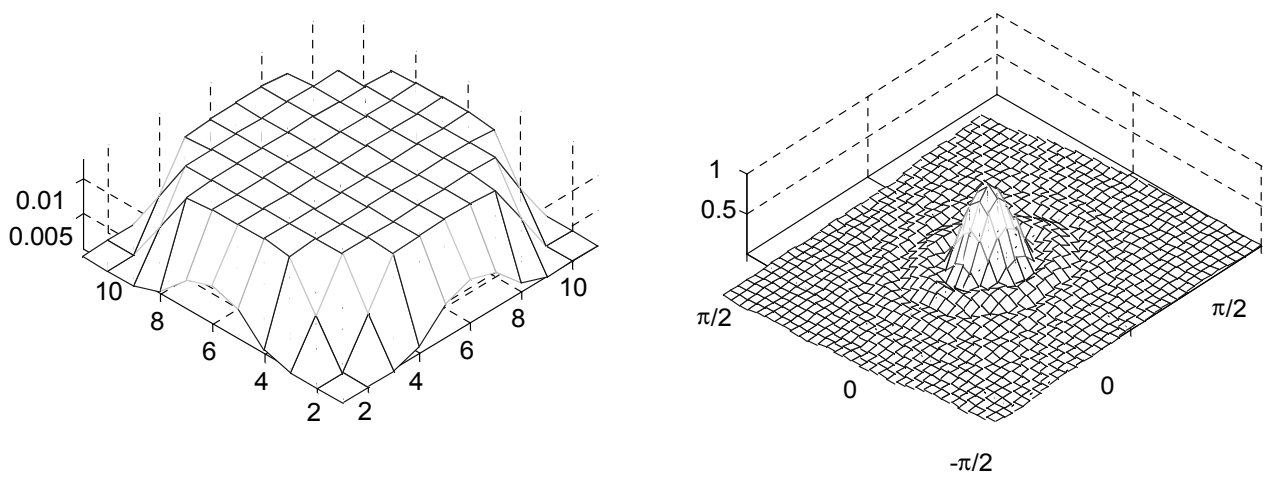

Fig. 6. PSF of uniform out of focus (circular) blur (with $R=5$ ) and its Fourier transform (magnitude).

of the kernel function. Convert the image to spatial domain and calculate its nongaussianity for different population samples.

4. Generate the child population for next iteration by evolving from the parents on the basis of the fittest function (that is, the nongaussianity measure), and optimize the fitness function in subsequent iterations (generations).

5. Go back to Step (3) till a convergence criterion is met or a pre-set maximum number of generation (e.g. 50) is reached.

The proposed method is computationally simple and easy to implement. It can be applied for estimation of the parameters of the blurring filter such as the spread of Gaussian blurring filter or length of motion in the case of motion blur. Furthermore, different PSFs are applied for the blind deconvolution of the blurred images with and without noise. The proposed algorithm has been tested on various image degradation functions.

\subsection{Atmospheric turbulence Blur}

Atmospheric turbulence is a severe limitation in remote sensing. Although the blur introduced by at- mospheric turbulence depends on a variety of factors (such as temperature, wind speed, exposure time), for long-term exposures the point-spread function can be described reasonably well by a Gaussian function:

$$
b(i, j ; \sigma)=C \exp \left(-\frac{i^{2}+j^{2}}{2 \sigma^{2}}\right) .
$$

Here $\sigma$ determines the amount of spread of the blurring, and the constant $C$ is to be chosen so that energy conservation rule is satisfied. The discrete version of Eq. (10) is usually obtained by numerical discretization of the continuous PSF for each element. Since the spatially continuous PSF does not have a finite support, it has to be truncated properly. Figure 4 shows such a PSF and its similar shape in the spectral domain (with $\sigma=$ 2). Note that Gaussian blurs do not have exact spectral zeros.

The proposed method has been applied for deblurring images of atmospheric (Gaussian) blurring. Figure 5 displays the result for the Cameraman image: the blurred image (blurred with Gaussian PSF with $\sigma=3$ ) and the estimated, blind-deblurred image by the proposed method. The estimated blurring parameter is $\sigma=2.91$ ). 


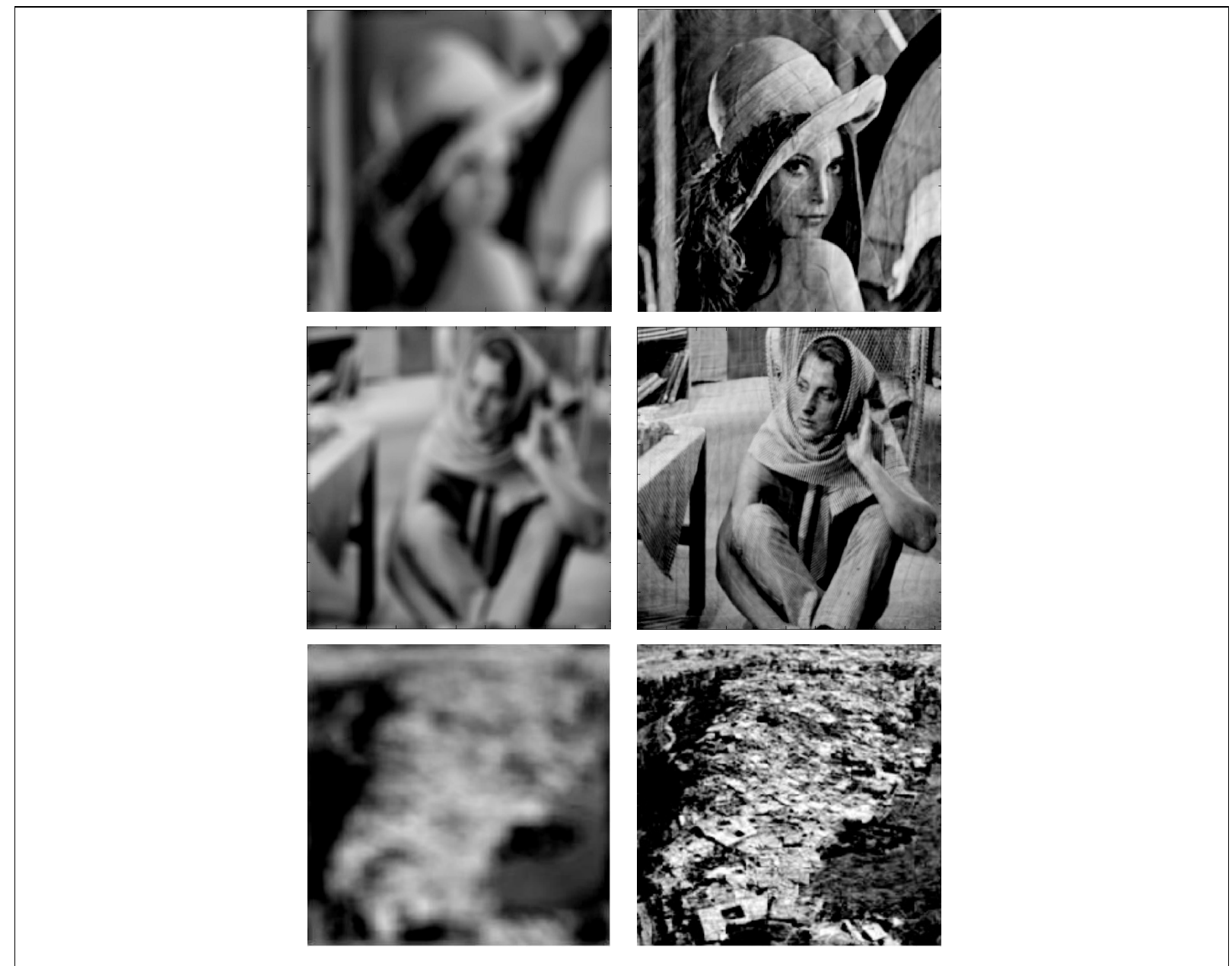

Fig. 7. Left: out of focus blurred images of radius 11, and right: restored with estimated radius 11.15, 11.20 and 11.23, on Lena, Barbra and an earthquake images, respectively.
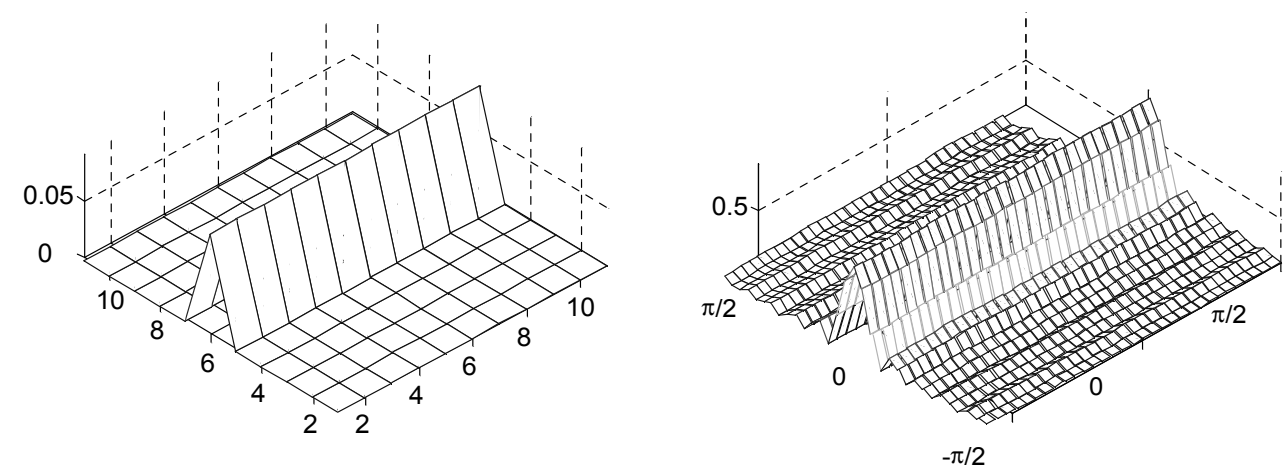

Fig. 8. PSF of linear motion blur of length 11 and angle 0 degree, and its Fourier transform.

\subsection{Uniform out of focus blur}

When a camera takes a 3-D scene onto a 2-D imaging plane, some parts of the scene are in focus white other parts may not be. If the aperture of the camera is circular, the image of any point source is a small disk, known as the circle of confusion (COC). The degree of defocus (diameter of the COC) depends on the focal 
PSNR comparison of the proposed method with other techniques. Note that in Wiener and Regularised filters and both Richardson-Lucy methods, the true PSF was supplied as the initial PSF. So their results can only be interpreted the upper limits. When a random initial PSF was used they do not converge to good results

\begin{tabular}{|c|c|c|c|c|c|c|c|}
\hline & \multirow[b]{2}{*}{$\begin{array}{l}\text { Blurring } \\
\text { width } \sigma\end{array}$} & \multicolumn{5}{|c|}{ PSNR (dB) } & \multirow[b]{2}{*}{$\begin{array}{c}\text { Proposed } \\
\text { method }\end{array}$} \\
\hline & & $\begin{array}{c}\text { Blurred and } \\
\text { noisy image }\end{array}$ & Wiener filter & $\begin{array}{l}\text { Regularized } \\
\text { filter }\end{array}$ & $\begin{array}{l}\text { Richardson- } \\
\text { Lucy method }\end{array}$ & $\begin{array}{l}\text { Blind Richardson- } \\
\text { Lucy method }\end{array}$ & \\
\hline \multirow[t]{4}{*}{ Barbra } & 2 & 23.56 & 23.72 & 23.72 & 23.40 & 22.96 & 24.08 \\
\hline & 3 & 22.82 & 23.01 & 23.01 & 23.46 & 23.36 & 23.68 \\
\hline & 4 & 22.23 & 22.41 & 22.41 & 23.19 & 23.17 & 23.33 \\
\hline & 5 & 21.81 & 21.99 & 21.98 & 22.87 & 22.86 & 22.97 \\
\hline \multirow[t]{4}{*}{ Lena } & 2 & 27.68 & 28.01 & 28.01 & 27.83 & 27.03 & 29.63 \\
\hline & 3 & 25.66 & 25.66 & 25.66 & 27.72 & 27.56 & 28.02 \\
\hline & 4 & 24.40 & 24.57 & 24.57 & 26.78 & 26.74 & 26.97 \\
\hline & 5 & 23.61 & 23.76 & 23.76 & 25.70 & 25.70 & 25.93 \\
\hline \multirow[t]{4}{*}{ Boat } & 2 & 25.71 & 25.64 & 25.64 & 26.82 & 26.28 & 27.42 \\
\hline & 3 & 23.87 & 23.75 & 23.75 & 25.95 & 25.87 & 26.01 \\
\hline & 4 & 22.81 & 22.73 & 22.73 & 24.77 & 24.77 & 23.80 \\
\hline & 5 & 22.16 & 22.14 & 22.14 & 23.71 & 23.71 & 22.73 \\
\hline \multirow[t]{4}{*}{ Zelda } & 2 & 30.91 & 32.13 & 32.13 & 28.37 & 25.85 & 32.85 \\
\hline & 3 & 29.06 & 30.09 & 30.09 & 29.72 & 28.70 & 31.40 \\
\hline & 4 & 27.83 & 28.69 & 28.69 & 29.63 & 29.25 & 30.42 \\
\hline & 5 & 26.99 & 27.81 & 27.81 & 28.96 & 28.80 & 29.47 \\
\hline
\end{tabular}

length, the aperture value of the lens, and the distance between the camera and object. An accurate model would describe the diameter of the COC and provide the intensity distribution within the COC. However, if the degree of defocusing is large relative to the wavelengths considered, a geometrical approach can be followed resulting in a uniform intensity distribution within the COC. The spatially continuous PSF of this uniform out-of-focus blur with radius $R$ is given by:

$$
b(i, j ; R)=\left\{\begin{array}{l}
\frac{1}{C \pi R^{2}} \text { if } \sqrt{i^{2}+j^{2}} \leqslant R^{2} \\
0 \text { elsewhere }
\end{array},\right.
$$

where $C$ is a constant that must be chosen so that energy conservation law is satisfied. The approximation of Eq. (11) is incorrect for the fringe elements of the point-spread function. A more accurate model for the fringe elements would involve the integration of the area covered by the spatially continuous PSF that needs to be calculated by integration. Figure 6(a) shows the profiles of such a PSF and its Fourier transform.

Figure 6(b) shows the modulus of the Fourier transform of this type of PSF for $R=5$. Again a low pass behaviour can be observed (in this case both horizontally and vertically), as well as a characteristic pattern of spectral zeros. Figure 7 displays the results of applying the proposed method on this type of burring. Here the original images were blurred with uniform out of focus blur of radius 11. The proposed method was applied to optimize the parameters of the deblurring function by using kurtosis as the nongaussianity measure.
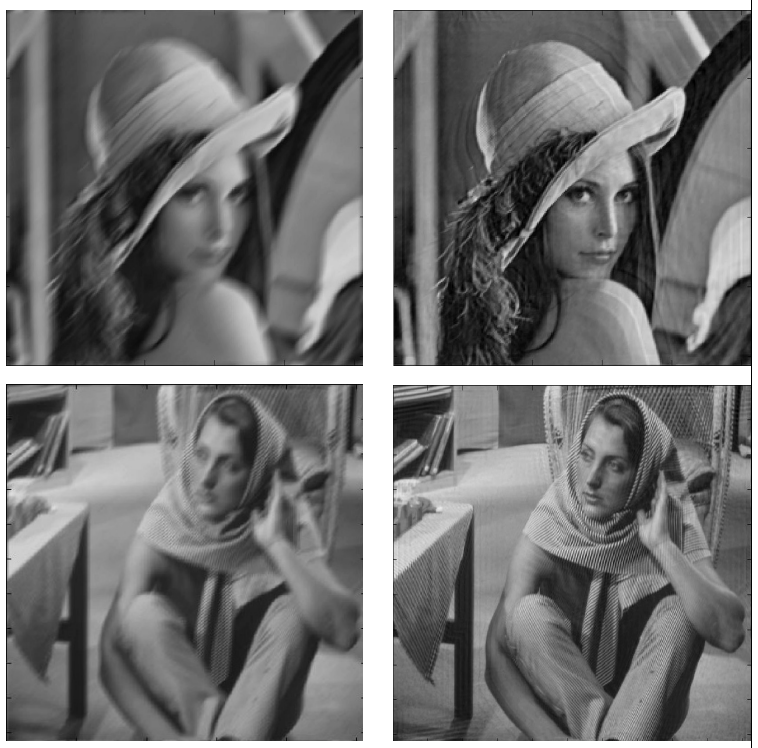

Fig. 9. Left: linear motion blurred images of length 11 and angle 45 degree, and right: restored images with estimated length of linear blur being 11.1 and 11.24 for Lena and Barbra, respectively.

\subsection{Linear motion blur}

Many types of motion blur are due to relative motion between the recording device and the scene. This can be in the form of a translation, a rotation, a sudden change of scale, or some combination of these. Here only the important case of a global translation is considered. When the scene to be recorded translates relative to the camera at a constant velocity under an angle of $\varphi$ 


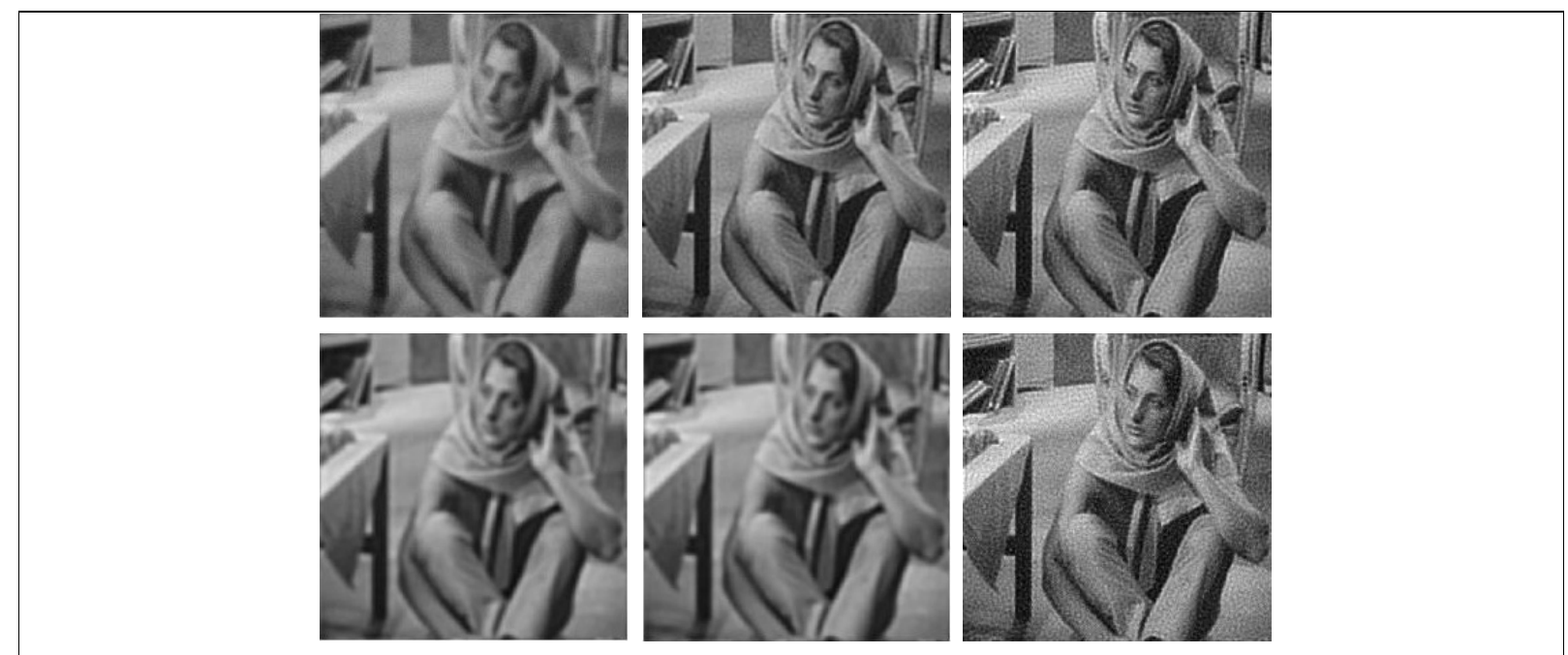

Fig. 10. Blurred and noisy Barbra image (top left), processed with the proposed method (top middle), the Richardson-Lucy (top right), Regularized filter (bottom left), Wiener Filter (bottom middle) and the Blind Richardson Lucy method (bottom right).
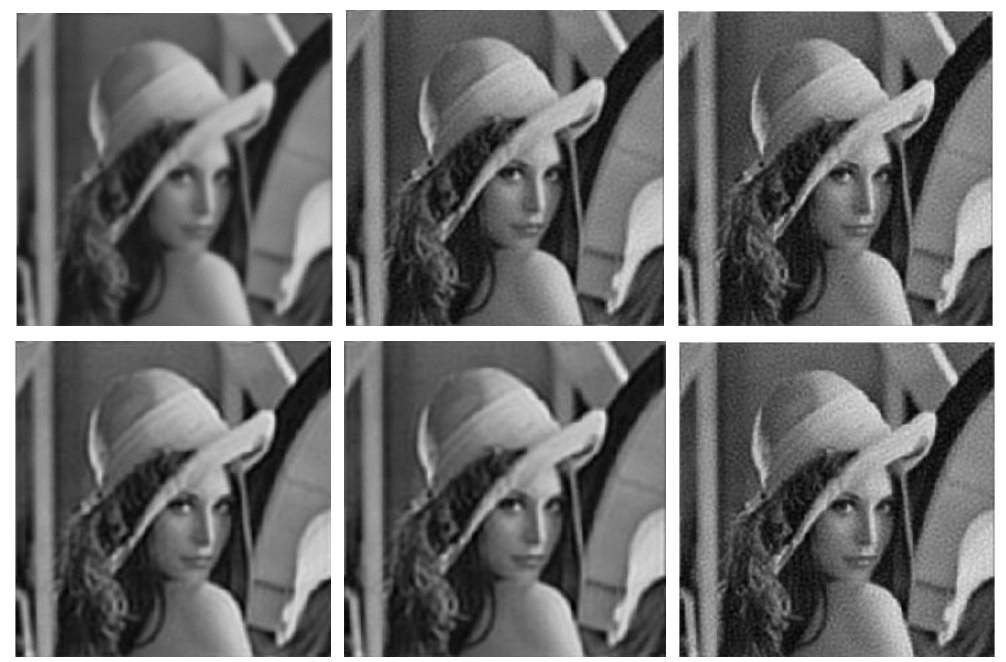

Fig. 11. Blurred and noisy Lena images (top left), processed with the proposed method (top middle), processed with Richardson Lucy (to right), processed with Regularized filter (bottom right), processed with Wiener Filter (bottom middle) and processed with Blind Richardson Lucy method (bottom right).

radians with the horizontal axis during the exposure interval, the distortion is one-dimensional. Denoting the length of motion by $L$, the PSF is given by:

$$
b(i, j ; L, \varphi)=\left\{\begin{array}{l}
\frac{1}{L} \text { if } \sqrt{i^{2}+j^{2}} \leqslant \frac{L}{2} \text { and } \frac{i}{j} \\
=-\tan \varphi \\
0 \text { elsewhere }
\end{array}\right.
$$

Figure 8 shows the PSF of Eq. (12) for linear motion for length of 11 and angle of zero with the horizontal direction and its Fourier transform (magnitude).

The proposed method has been tested to estimate the blurring parameters for the subsequent deblurring by this PSF. In this case there are two parameters that are needed to be optimized, the length of motion and the angle of motion. In Fig. 9, results are shown for the degraded images and deblurring results.

\subsection{Deblurring and denoising}

The proposed method can also be extended to include denoising capability. The blurred and noisy (for example, by additive white Gaussian noise) image becomes more Gaussian compared to the original image. That is, its kurtosis or negentropy becomes more Gaussian as a 
result of the blurring and adding noise. Wiener filtering can be used to denoise and deblur the image; while the proposed algorithm is used to estimate the required parameters of the blurring PSF and the noise. The algorithm is able to approximate the parameters and subsequently to denoise and deblur the image. Typical results of such experiments are shown in Figs 10 and 11, where images were blurred with Gaussian blurring function and then added with Gaussian white noise.

A comparison of the proposed method with other existing techniques (such as Wiener filter, regularized filter, Richardson-Lucy method and blind Richardson Lucy method) has been carried out on several benchmark images such as Barbra, Lena, Boat and Zelda. They were evaluated on both qualitatively visual quality and PSNR (peak signal-noise ratio) measures. PSNR performances on these images are given in Table 1. The proposed method produces markedly superior results not only in terms of improved visual quality but also in quantitative PSNR terms over the existing methods. Note that the true PSF was supplied to the Wiener filter, regularization filter and the Richardson-Lucy techniques as they are not blind methods. Even for the blind Richardson-Lucy method, it requires good a priori knowledge of the PSF as the initial estimate (the true PSF was used in this case). The proposed blind method however operates in a truly blind manner and still outperforms these existing methods in almost all cases.

\section{Conclusions}

A new method based on ICA, nongaussianity measures and genetic algorithms for blind deconvolution and deblurring of signals and images has been proposed. The proposed method is computationally simple and easy to implement in practice and most important of all, it is completely blind and works on a single image. The method is conducted in spectral domain where nongaussianity analysis and genetic algorithm are used for optimizing the parameters of the candidate degrading functions. The method does not require any a priori knowledge about either the image or the blurring function. The proposed method has been tested on a number of images blurred with various PSFs with and without additive noise. The results indicate that the proposed method is able to deblur images effectively. A comparison with the existing deconvolution and deblurring methods shows the superior performance of the proposed blind method over the others even when prior knowledge of the PSF is given to these methods. Future endeavours are to tackle heavy noise and robustness against outliners as well as to improve on the efficiency of the parameter optimization processes.

\section{Acknowledgement}

We would like to thank anonymous reviewers for their helpful and constructive comments, which have helped the revision of the paper enormously.

\section{References}

[1] G.R. Ayers and J.C. Dainty, Iterative blind deconvolution method and its applications, Optics Letters 13 (1988), $547-$ 549.

[2] M.R. Banham and A.K. Katsaggelos, Digital image restoration, IEEE Signal Processing Magazine 14(2) (1997), 24-41.

[3] D.A. Fish, A.M. Brinicombe, E.R. Pike and J.C. Walker, Blind deconvolution by means of the Richardson-Lucy algorithm. Journal of the Optical Society of America A 12 (1995), 58-65.

[4] R.C. Gonzalez and R.E. Woods, Digital Image Processing, Prentice Hall, 2002.

[5] A. Hyvärinen, Survey on independent component analysis. Neural Computing Surveys 2 (1999), 94-128.

[6] A. Hyvärinen, J. Karhunen and E. Oja, Independent Component Analysis, John Willey \& Sons, 2001.

[7] A. Jalobeanu, L.B. Feraud and J. Zerubia, An adaptive Gaussian model for satellite image deblurring, IEEE Trans. Image Processing 13 (2004), 613-621.

[8] D. Kundur and D. Hatzinakos, Blind image deconvolution, IEEE Signal Processing Magazine 13(3) (1996), 43-64.

[9] R.L. Lagendijk and J. Biemond, Basic methods for image restoration and identification, in: Handbook of Image and Video Processing, A.C. Bovik and J.D. Gibson, eds, 2000.

[10] L.B. Lucy, An iterative technique for the rectification of observed images, Astronomical Journal 79 (1974), 745-754.

[11] N. Moayeri and K. Konstantinides, An Algorithm for blind restoration of blurred and noisy images, Hewlett Packard Labs Technical Report HPL-96-102, available at http://www.hpl.hp.com/techreports.

[12] T.F. Rabie, R.M. Rangayyan and R.B. Paranjape, Adaptiveneighborhood image deblurring, Journal of Electronic Imaging 3 (1994), 368-378.

[13] W.H. Richardson, Bayesian-based iterative method of image restoration, Journal of the Optical Society of America 62 (1972), 55-59.

[14] S. Umeyama, Blind deconvolution of blurred images by use of ICA, Electronics and Communications in Japan Part III, 84 (2001), 1-9.

[15] C. Vural and W.A. Sethares, Blind Deconvolution of Noisy Blurred Images Via Dispersion Minimization, 14th IEEE Int. Conf. on Digital Signal Processing, Santorini, Greece, 2002.

[16] R.L. White, Image restoration using the damped RichardsonLucy method, in: The Restoration of HST Images and Spectra - II, R.J. Hanisch and R.L. White, eds, 1994.

[17] H. Yin and I. Hussain, ICA and Genetic Algorithms for lind Signal and Image Deconvolution and Deblurring, Proc. International Conference on intelligent Data Engineering and Automated Learning (IDEAL'06), LNCS-4224, 2006, 595-603. 